\title{
Self-adhesive hydrogel meshes reduce tissue incorporation and mechanical behavior versus microgrips self-fixation: a preclinical study
}

\author{
Selma Benito-Martínez ${ }^{1,2,3} \cdot$ Marta Rodríguez $^{2,3,4} \cdot$ Francisca García-Moreno ${ }^{2,3,4} \cdot$ Bárbara Pérez-Köhler $^{1,2,3}$. \\ Estefanía Peña $^{2,5} \cdot$ Begoña Calvo ${ }^{2,5} \cdot$ Gemma Pascual ${ }^{1,2,3}$ (1) Juan Manuel Bellón ${ }^{2,3,4}$
}

Received: 20 October 2021 / Accepted: 11 December 2021 / Published online: 7 January 2022

(c) The Author(s) 2022

\begin{abstract}
Purpose Atraumatic mesh fixation for abdominal hernia repair has been developed to avoid the disadvantages of classical fixation with sutures, which is considered a cause of chronic pain and discomfort. This study was designed to analyze, in the short and medium term, the biological and mechanical behavior of two self-fixing meshes compared to that of a polypropylene (PP) mesh fixed with a cyanoacrylate (CA) tissue adhesive.

Methods Partial abdominal wall defects $(6 \times 4 \mathrm{~cm})$ were created in New Zealand rabbits $(n=36)$ and repaired using a selfadhesive hydrogel mesh (Adhesix ${ }^{\mathrm{TM}}$ ), a self-gripping mesh (ProGrip ${ }^{\mathrm{TM}}$ ) or a PP mesh fixed with CA (Surgipro ${ }^{\mathrm{TM}} \mathrm{CA}$ ). After 14 and 90 days, the host tissue incorporation, macrophage response and biomechanical strength were examined.

Results At 14 and 90 days, the ProGrip and Surgipro CA meshes showed good host tissue incorporation; however, the Adhesix implants presented poor integration, seroma formation and a higher degree of shrinkage. The Adhesix hydrogel was completely reabsorbed at 14 days, whereas ProGrip microhooks were observed at all study times. The macrophage response was higher in the ProGrip and Surgipro CA groups at 14 and 90 days, respectively, and decreased over time. At 90 days, the ProGrip implants showed the highest tensile strength values and the Adhesix implants showed the highest failure stretch. Conclusion Meshes with mechanical microgrip self-fixation (ProGrip) show better biological and mechanical behavior than those with adhesive hydrogel (Adhesix) in a preclinical model of abdominal hernia repair in rabbits.
\end{abstract}

Keywords Hernia repair $\cdot$ Self-adhesive meshes $\cdot$ Self-gripping meshes $\cdot$ Tissue integration $\cdot$ Biomechanical test

Gemma Pascual and Juan Manuel Bellón shared the senior authorship.

Gemma Pascual

gemma.pascual@uah.es

1 Departamento de Medicina y Especialidades Médicas, Facultad de Medicina y Ciencias de la Salud, Universidad de Alcalá, 28805 Alcalá de Henares, Madrid, Spain

2 Biomedical Networking Research Centre On Bioengineering, Biomaterials and Nanomedicine (CIBER-BBN), 28029 Madrid, Spain

3 Ramón y Cajal Health Research Institute (IRYCIS), 28034 Madrid, Spain

4 Departamento de Cirugía, Facultad de Medicina y Ciencias de la Salud, Ciencias Médicas y Sociales, Universidad de Alcalá, 28805 Alcalá de Henares, Madrid, Spain

5 Aragón Institute of Engineering Research (I3A), Universidad de Zaragoza, Zaragoza, Spain

\section{Introduction}

The implantation of prosthetic materials for the repair of hernial defects in the abdominal wall is one of the most frequent general surgical procedures [1]. Different meshes for hernia repair have evolved in recent years in terms of the structure and chemical composition [2-6]. However, polypropylene (PP) meshes continue to be the most often used material for abdominal hernia repair.

These PP reticular prostheses, in the surgical implant procedure, when necessary, are usually fixed to the surrounding tissues using penetrating devices, to prevent migration, which can lead to hernia recurrence.

The short-term strength of mesh fixation is an undescribed factor in hernia repair but could have significant implications for early recurrence and mesh contraction [7]. Numerous techniques are available for fixation, and the most frequently used classical fixation devices range from 
simple sutures to different stapling systems as well as tacks at the laparoscopic level [8-11]. These routinely used fixation devices have significantly reduced the recurrence rates for hernia repair; however, they are time-consuming and led to postoperative discomfort and chronic pain, which have a significant impact on health-related quality of life [12].

In this respect, many researchers recommend reducing or avoiding the use of these classical devices to reduce the traumatic effects at the tissue level; thus, tissue adhesives have become popular and prostheses with self-adhering properties have been developed [13-16].

Nonpenetrating mesh fixation technologies have many advantages, such as easy handling and speed of use, reducing operative time [17]. Specifically, any method of fixation that does not use sutures or tacks to penetrate the underlying tissue where it is implanted has continued to gain popularity because such methods minimize the tissue trauma caused by sutures when the needle or tack is inserted, which represents an important consideration, especially in anticoagulated patients [18]. Finally, these methods can prevent certain adverse effects, including irritation, entrapment or injury to nerve endings [13], which in some cases would explain the postoperative pain of patients undergoing hernioplasty [19-21].

Mesh-tissue interfacial strength can also be used as a marker of mesh-tissue integration in the long term or mesh-tissue adhesion in the short term. Either marker indicates the capacity of the mesh-tissue complex to brace or carry mechanical loading, which is a critical mechanism for absorbing the stresses that develop in the abdominal wall under physiological loading conditions in both the short and long term [7]. Therefore, these novel adhesive strategies must also be strong enough to provide adequate clamping force to prevent the mesh from becoming detached, mainly in the short term before host tissue ingrowth.

The most widely used tissue adhesives specifically for hernia repair are fibrins of biological origin [22, 23]; however, long-chain cyanoacrylate-type tissue adhesives, for which toxicity problems have been minimized, are increasingly being used and show excellent biocompatibility and mechanical behavior and are currently authorized for use in clinical practice $[24,25]$.

In the group of atraumatic fixation devices, two relatively novel interesting prostheses, which have not been very widely tested, incorporate absorbable microhooks (ProGrip) or adhesive hydrogel (Adhesix) into the traditional PP prosthesis, thus allowing for self-fixing without any other additional element; thus, their advantages may lead to promising outcomes [26, 28].

Taking into account all these aspects, the aim of the present preclinical study was to analyze the biological and mechanical behavior of these two self-fixing meshes relative to that of a PP mesh fixed using a synthetic adhesive (n-hexyl) cyanoacrylate (CA).

\section{Materials and methods}

\section{Experimental animals and meshes}

The maintenance of the animals used in this study and the experimental procedures were carried out in accordance with the current protocols on the use of animals in experimentation (European Directive 2010/63/EU, European Convention of the Council of Europe ETS123 and Spanish Royal Decree 53/2013), and the study was approved by the Animal Experimentation Ethics Committee of Universidad de Alcalá (Spain). In this study, partial defects were created in the abdominal wall in a total of 36 New Zealand white rabbits weighing $3 \mathrm{~kg}$, and the animals were divided into three groups according to the prosthesis used in the repair. Two study times at 14 and 90 days were established. Each of the groups had $n=6$ animals. The materials used to repair the defects were two self-fixing meshes or a Surgipro mesh fixed with a CA adhesive for medical use (Fig. 1):

Adhesix ${ }^{\mathrm{TM}}$ (Bard DAVOL Inc., Warwick, RI, USA) is a self-adhesive mesh composed of a PP mesh covered by a layer of polyethylene glycol and polyvinylpyrrolidone, and
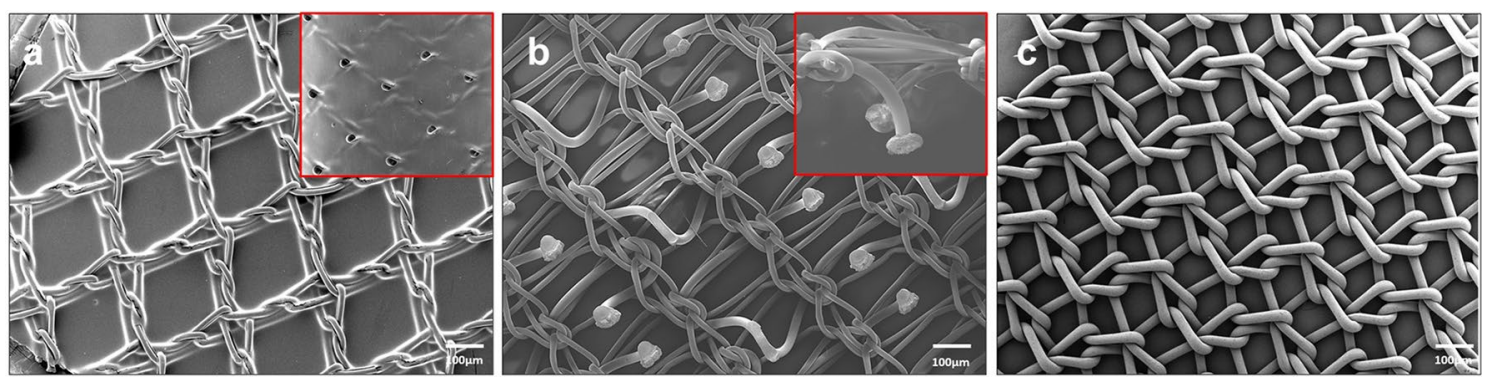

Fig. 1 Characterization of the prosthetic materials. Scanning electron microscopy (SEM) images $(15 \times$ magnification) of the Adhesix (a), Progrip (b) and Surgipro (c) meshes. Further magnified views of the reabsorbable layer of Adhesix (20x) and reabsorbable microhooks of Progrip (40x) are shown in the box 
in the presence of moisture, this layer forms a hydrogel that adheres to the tissue of the implant area and is reabsorbed within 7 days of implantation according to the manufacturer's specifications.

Progrip $^{\mathrm{TM}}$ (Medtronic, Minneapolis, MN, USA). A selfgripping mesh composed of PP with reabsorbable polylactic acid microhooks that provide immediate fixation to the implant tissue and are reabsorbed within 12-15 months.

Surgipro $^{\mathrm{TM}}$ CA. PP mesh (Medtronic) fixed with $150 \mu \mathrm{l}$ of CA adhesive (Ifabond, Peters Surgical, Bobigny, France) was applied as a spray with a diffuser (Sample Spray 2.5; Sunbox Distribution, Barcelona, Spain).

\section{Surgical technique and sample collection}

Before surgery, the animals received an analgesic dose of $0.05 \mathrm{mg} / \mathrm{kg}$ buprenorphine (Buprecare, Divasa Farmavic, Barcelona, Spain). Subsequently, general anesthesia was induced with ketamine $(20 \mathrm{mg} / \mathrm{kg}$, Imalgene, Merial, Barcelona, Spain) and xylazine (3 mg/kg, Xilagesic $2 \%$, Calier, Barcelona, Spain) administered intramuscularly. Using a sterile surgical technique, an approximately 7 -cm-long incision in the skin was made in the right lateral side at a $3 \mathrm{~cm}$ distance from the abdominal linea alba.

The skin was dissected from the abdominal wall to leave a surgical field $(6 \times 4 \mathrm{~cm})$ that allowed for the excision of the internal and external oblique muscle, leaving intact the transverse muscle, fascia transversalis and parietal peritoneum. In a random manner, each partial defect was repaired using $6 \times 4 \mathrm{~cm}$ meshes. No additional fixation methods described in the study group were used. The subcutaneous tissue and skin were closed with a running $3 / 0$ suture (Ethicon, Somerville, NJ, USA). After surgery and once daily for the first 3 days postsurgery, the animals received an analgesic dose of buprenorphine. Following surgery, the animals were monitored daily to detect any signs of postsurgical complications (warmth, dehiscence, abscess formation, etc.). 14 and 90 days post-implantation, the animals were anesthetized again and euthanized with an overdose of intravenous sodium pentobarbital (150 mg/kg, Dolethal, Vétoquinol, Lure, France).

At the end of the established study times, the dimensions of the meshes were measured and photographed before excision from the abdominal wall to determine whether there was a reduction in the implant area with respect to the original size meshes ( $\%$ mesh shrinkage). The percentage of shrinkage was determined by computerized image analysis using ImageJ software (https://imagej.nih.gov/ij).

From each of the animals, skin and subcutaneous tissue were removed and specimens that included both mesh and surrounding tissue were collected. Two or three (depending on the degree of implant shrinkage) tissue and mesh samples of $1.5 \mathrm{~cm}$ were reserved for biomechanical tests. The rest of the sample was used for morphological and immunohistochemical studies.

\section{Morphological analyses}

For the light microscopy (LM) analyses, samples were fixed in F13 solution and embedded in paraffin, cut into 5-mmthick sections by microtome and stained according to the standard procedure for hematoxylin-eosin, Masson trichrome and Sirius red. Finally, the samples were mounted using Canada balsam and then visualized with a Zeiss Axiophot microscope (Carl Zeiss, Oberkochen, Germany). Sirius red staining was observed under polarized light. This technique is based on the orientation and interaction between the sulfone groups of the dye, the amine groups of lysin and hydrolysin, and the guanidine groups of arginine in the collagen fibers, thus producing different colors depending on the type of collagen. Type I collagen (mature) appears as a reddish-orange stain, while type III collagen (immature) takes on a yellowish-green shade.

Tissue fragments were fixed in 3\% glutaraldehyde for scanning electron microscopy (SEM), dehydrated in a graded series of ethanol, desiccated with carbon dioxide using a Polaron CPD7501 critical point dryer (Fisons Instruments, Glasgow, UK), gold-palladium coated, and examined under a JSM-IT500 InTouchScope ${ }^{\mathrm{TM}}$ scanning electron microscope (JEOL Ltd., Tokyo, Japan).

\section{Immunohistochemical analyses}

For the immunohistochemical study, the macrophages number per microscopic field was determined after labeling with the rabbit monoclonal antibody RAM-11 (DAKO M-633, Santa Clara, CA, USA; dilution 1:50) using a conventional protocol for avidin-biotin procedures. Biotinated $\mathrm{IgG}$ was used as a secondary antibody to amplify the signal and then labeled with avidin. To detect the antigen-antibody reaction, an alkaline phosphatase-fast red reaction was used, followed by final cell nuclei staining with hematoxylin. RAM-11-positive cells were counted in 10 microscopy fields per sample using a Zeiss Axiophot microscope (magnification 200×).

\section{Biomechanical assay}

For mechanical testing, the strength of mesh fixation to the underlying tissue was evaluated at 14 and 90 days using the lap-shear method on freshly harvested samples immediately after slaughter according to the standard F2255-05 (standard test method for strength properties of tissue adhesives in lap-shear by tension loading). The strip length, width and thickness were determined with a digital caliper. Three measurements at different locations were obtained for each sample to ensure sample homogeneity. One short edge of 
the mesh was freed from the fascia at a length of $1 \mathrm{~cm}$ and inserted between the instrument's upper clamps, thus leaving $3 \mathrm{~cm}$ adhered to the tissue (fixation testing area). The bottom clamp clasped the abdominal wall tissue. Tests were performed under displacement control on an INSTRON 3340 tensiometer (Instron Corp., Norwood, MA, USA) with a 1 $\mathrm{kN}$ full-scale load cell. The applied displacement rate was $5 \mathrm{~mm} / \mathrm{min}$ until failure. The load (maximum load sustained) and displacement at failure were recorded. To compare the adhesion strength of the different groups, we defined two new variables: tensile strength, which was computed as $P$ $[\mathrm{N} / \mathrm{mm}]=$ load $[N] /$ width $[\mathrm{mm}]$; and failure stretch, which was computed as $\lambda[-]=L_{0}+\Delta L / L_{0}$, where $\mathrm{L}_{0}$ is the initial distance between the clamps and $\Delta L$ is the displacement.

\section{Statistical analysis}

The statistical comparison of the data (mean and standard deviation) was performed using Student's paired $t$ test (in the case of a normal distribution) or Wilcoxon's test (in the case of a nonnormal distribution). The test of normality was performed by the Shapiro-Wilk test, and $p$ values less than 0.05 were considered significant (no normal distribution). Statistical analyses were performed using MATLAB 2010 (The MathWorks Inc., Natick, MA, USA).

\section{Results}

\section{Macroscopic observations}

There were no complications related to the anesthesia or surgical procedures. No signs of surgical site or mesh infection were observed in any of the three study groups; therefore, all animals were included in the study.

At 14 days post-implantation, seroma was evident in the Adhesix implants (four of six implants: 4/6) and some Surgipro CA implants (2/6). All of them showed a thin fibrous capsule. Contrary to these observations, animals from the Progrip group did not exhibit any seroma formation. At 90 days post-implantation, seroma formation was not evident in any of the groups.

Adhesix exhibited poor integration into host tissue after 14 days of implantation. Three of six implants (3/6) showed small areas where the mesh was detached from the host tissue. Such detachment was not observed in any of the Progrip and Surgipro CA samples, where the meshes presented good tissue integration.

At 90 days postoperative, poor tissue integration was also observed in two of six (2/6) Adhesix implants while good integration into the host tissue was observed for the Progrip and Surgipro CA implants (Fig. 2a).
Prior to sample collection, photographs were taken and the prosthetic material was measured to determine the shrinkage in the implant area after the repair process.

At 14 days, the macroscopic results and implant area measurements revealed that the Adhesix implants showed a significantly higher degree of shrinkage than the Progrip and Surgipro CA implants $(p<0.05)$. The lowest values of shrinkage were observed for Progrip, and the values were significant relative to that of Surgipro CA $(p<0.05)$. At 90 days, there was a slight increase in the shrinkage percentage in all groups, although Adhesix again showed values significantly higher than that of the other two groups $(p<0.05)$ (Fig. 2b).

\section{Morphological analysis}

At 14 days, the histological findings of the Adhesix samples showed the presence of PP filaments; however, the hydrogel was completely reabsorbed. In some detached zones, the LM and SEM microscopy images allowed for the validation of macroscopic observations regarding the lack of mesh integration and seroma formation beneath the prosthetic materials (Fig. 3a-c).

Scar tissue was composed of collagen fibers, predominantly type III collagen (Table 1), and inflammatory cells, including macrophages and foreign body giant cells, appeared around filaments and more numerous in areas associated with seroma.

At 90 days post-implantation, the inflammatory process had resolved, repair connective tissue richer in type I collagen was observed around the prosthetic filaments (Table 1), and the space between the mesh and underlying fascia was mostly occupied by adipose tissue (Fig. 3d-f).

At 14 and 90 days post-implantation, Progrip implants were fully integrated into the host tissue. Neoformed connective tissue showed the same distribution as Adhesix implants. Blood vessels, fibroblasts and type III collagen fibers (Fig. 4; Table 1) were observed. Polylactic acid microhooks were observed at all study times without signs of evident absorption. Macrophages and inflammatory cells, mostly monocytes and polymorphonuclear cells, were observed around the Progrip filaments and microhooks. Similar to the Adhesix implant, great development of adipose tissue beneath the prosthetic material was observed at 90 days postimplant (Fig. 4d-f).

In the Surgipro CA group, remnants of tissue adhesive were observed to be zones with different sizes dispersed between polypropylene filaments at 14 and 90 days (Fig. 5). Scar tissue was mainly distributed around the mesh filaments. However, in this group, this tissue was composed of larger amounts of collagen type I (Table 1). Fibroblasts, macrophages and other inflammatory cells were observed around filaments and adhesive margins. This behavior could 
Fig. 2 Macroscopic appearance of the Adhesix (a-c), Progrip (d-f) and Surgipro (g-i) meshes after implantation into the experimental animals $(\mathbf{a}, \mathbf{d}, \mathbf{g})$ at $14(\mathbf{b}, \mathbf{e}, \mathbf{h})$ and 90 days $(\mathbf{c}$, f, i) after surgery. The implant contour is indicated with a black dotted line. Percentage of shrinkage at the different study times (j). ${ }^{*} p<0.05$ (color figure online)
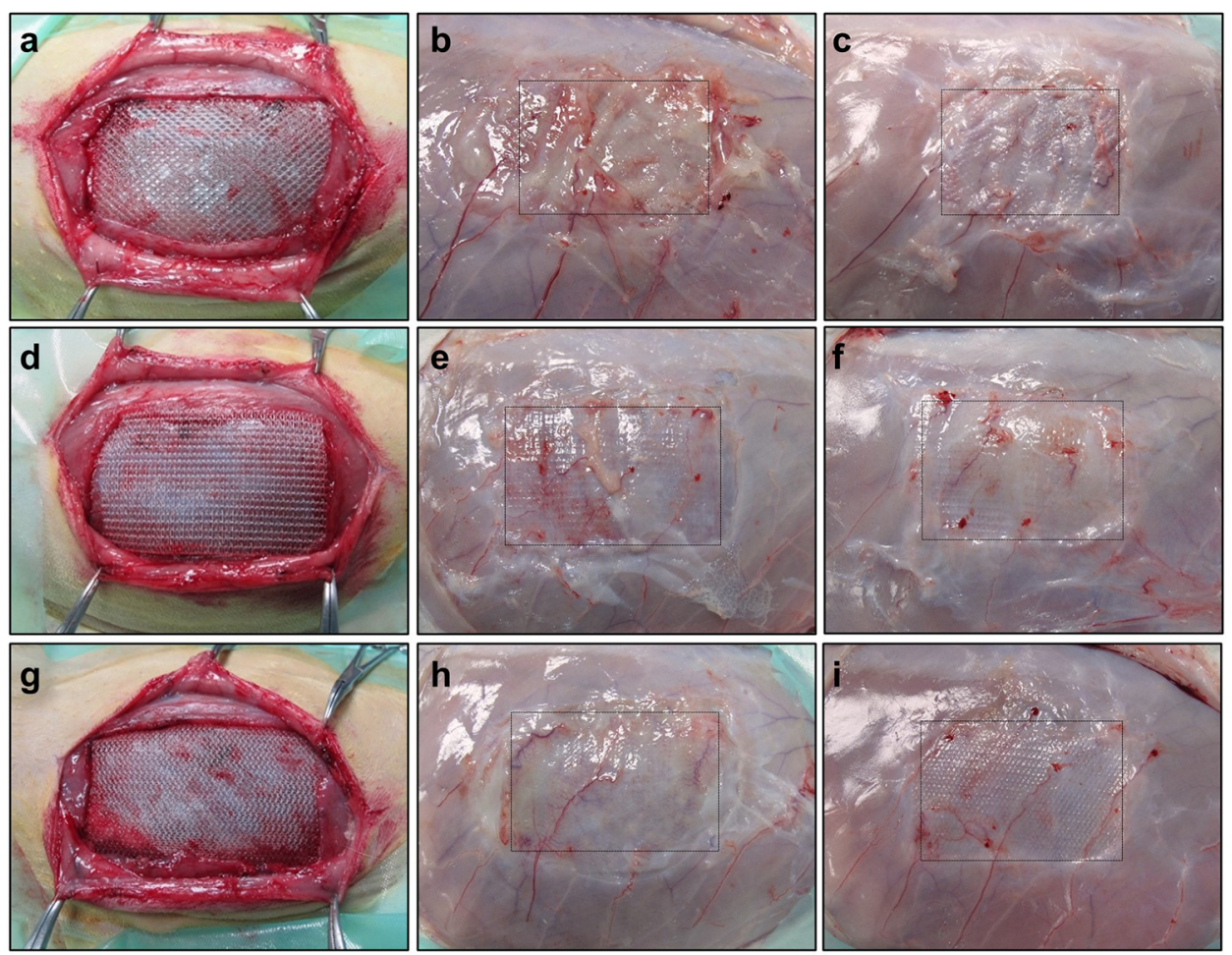

j

$*$
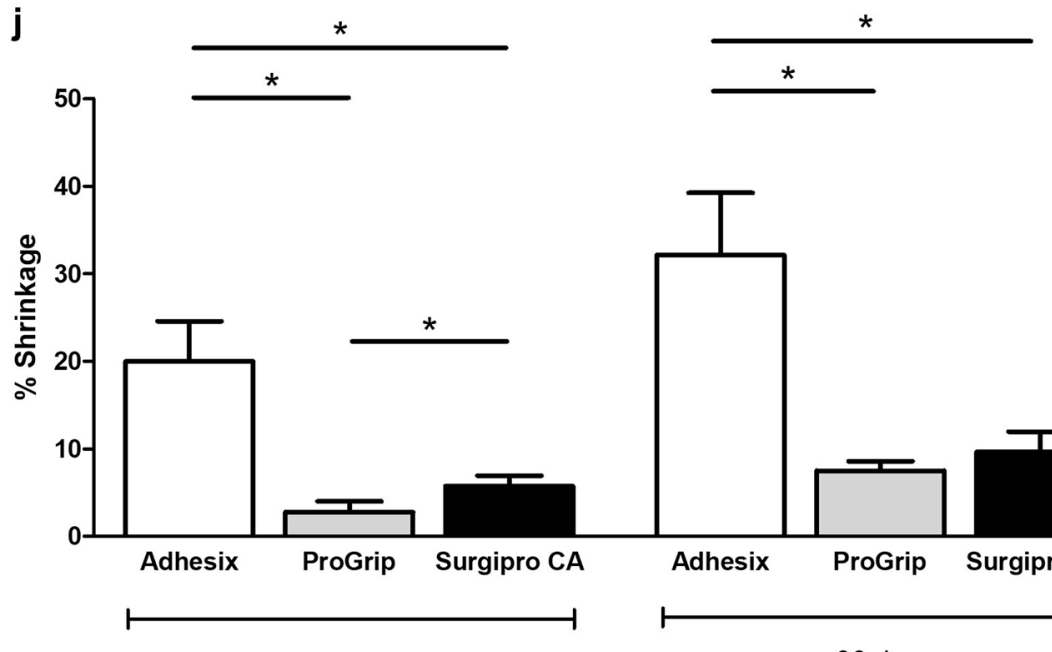

14 days 

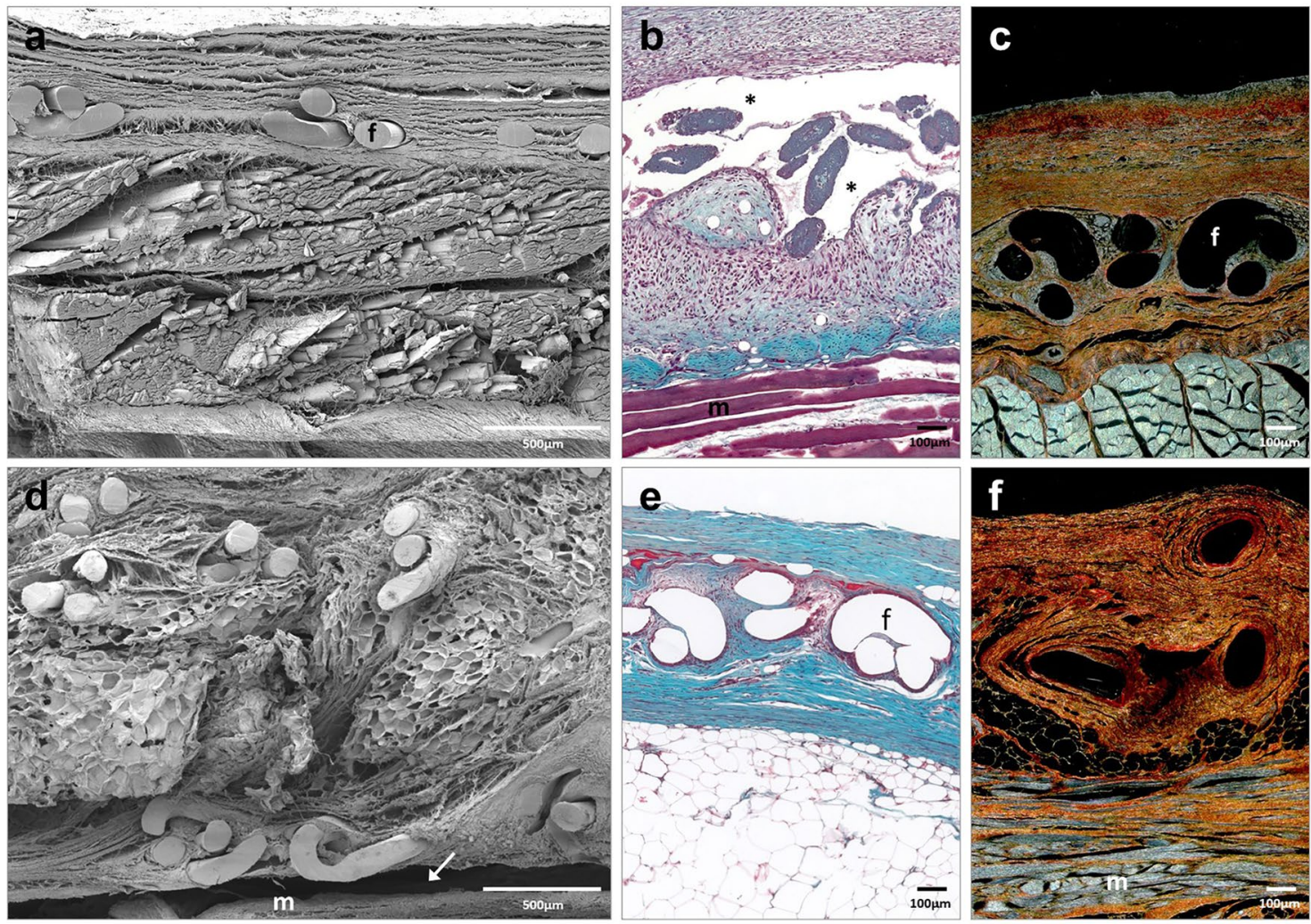

Fig. 3 Scanning electron microscopy (a, d) $(\times 500$; scales: $500 \mu \mathrm{m})$ and light microscopy images (Masson trichrome (b, e) and Sirius red staining $(\mathbf{c}, \mathbf{f}), \times 100$; scale: $100 \mu \mathrm{m})$ of Adhesix mesh at 14 days (a-c) and 90 days (d-f) post-implantation. Sirius red staining shows

Table 1 Semiquantification of collagen I (mature) and III (immature) expression in the neoformed tissue detected in the implant area in the different study groups

\begin{tabular}{llllll}
\hline & \multicolumn{2}{l}{ Collagens } & & \\
\cline { 2 - 3 } & 14 days & & & 90 days & \\
\cline { 2 - 3 } \cline { 5 - 6 } & Type I & Type III & & Type I & Type III \\
\hline Adhesix & + & +++ & & ++ & ++ \\
ProGrip & + & +++ & & ++ & ++ \\
Surgipro CA & ++ & ++ & & ++ & ++ \\
\hline
\end{tabular}

The scale used for semiquantification was as follows: + , minimum staining $(<25 \%) ;++$, moderate staining $(25-50 \%) ;+++$, strong staining (50-75\%); and ++++ , maximum staining $(>75 \%)$

the Progrip group $(p<0.05)$. At this time point, Adhesix showed significant differences with respect to Surgipro CA $(p<0.05)$ (Fig. 6g).

collagen I (mature) in red and collagen III (immature) in yellow. Symbols: f mesh filaments; $(\mathrm{m})$ muscle; $(\rightarrow)$ poor integration; and $(*)$ area of seroma (color figure online)

\section{Biomechanical study}

At 14 days post-implantation, the biomechanical analysis was not possible because $50 \%$ of the meshes in the Adhesix group were partially detached from the host tissue and 66\% presented seroma formation beneath the prosthetic material.

In the lap-shear methods, nonlinear behavior was observed regardless of the fixation method studied. In the statistical analysis, all fixation methods showed a normal distribution $(p>0.05)$.

At 90 days, significant differences in tensile strength and failure stretch values were observed between all fixation methods $(p<0.05)$. The highest tensile strength values were recorded in the Progrip group $(2.920 \pm 0.471 \mathrm{MPa})$, and the lowest values were obtained in the Adhesix group $(1.542 \pm 0.382 \mathrm{MPa})$. Statistical differences between both groups were observed $(p=0.0001)$. Failure stretching showed significant differences between the Adhesix group and the rest of the groups (Fig. 7b). 

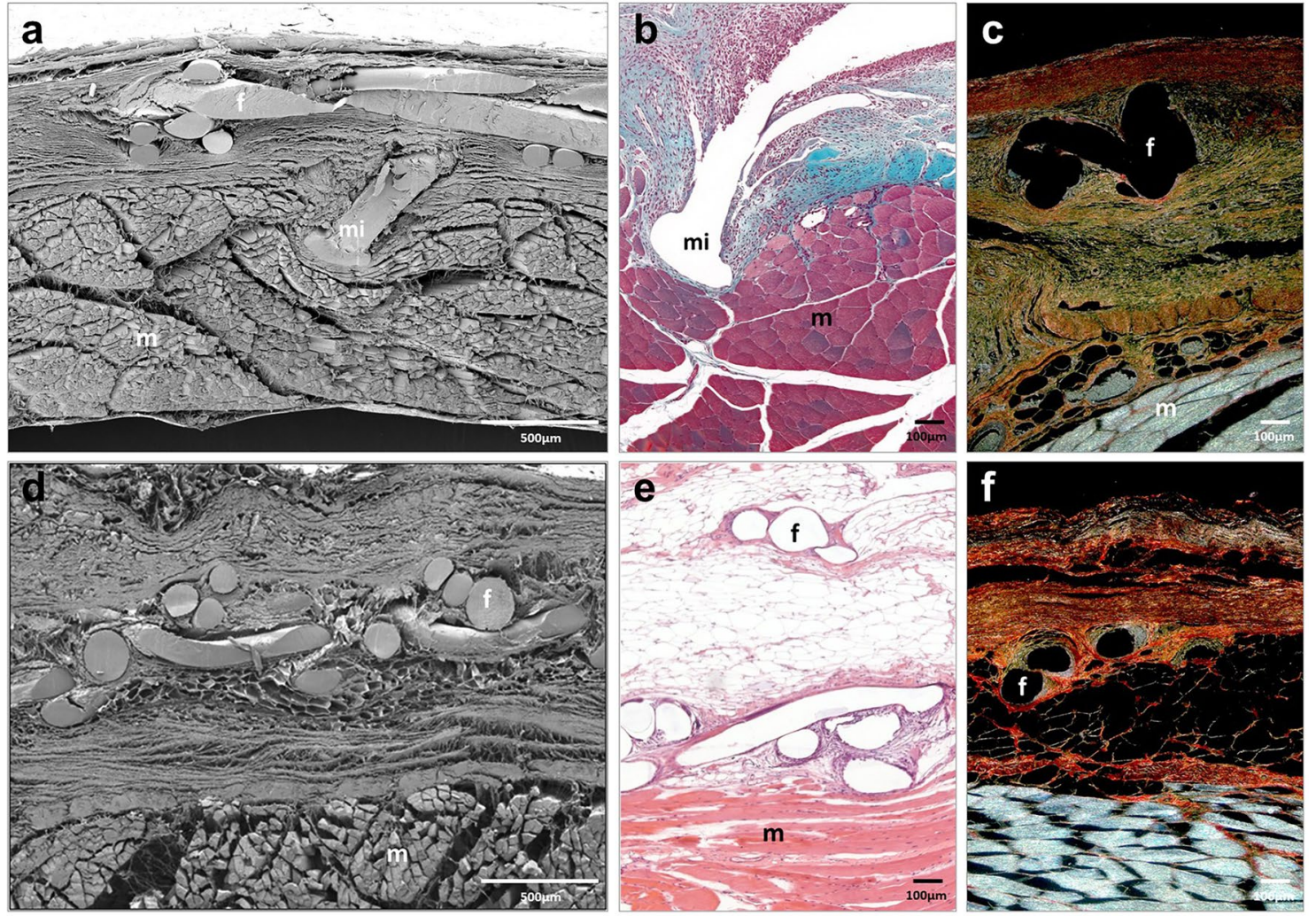

Fig. 4 Scanning electron microscopy $(\mathbf{a}, \mathbf{d})(\times 500$; scales: $500 \mu \mathrm{m})$ and light microscopy images (Masson trichrome (b), hematoxylin and eosin (e) and Sirius red staining $(\mathbf{c}, \mathbf{f}), \times 100$; scale: $100 \mu \mathrm{m})$ of Pro-

\section{Discussion}

In hernia repair processes on the abdominal wall, reinforcement with prosthetic materials in the form of meshes is considered the gold standard. In those cases in which the hernia defect is closed, mesh fixation would not be necessary, but otherwise prosthetic fixation is recommended to prevent the mesh from displacement in the early postoperative course. Currently, there are surgeons who leave these defects open ensuring that a 5-cm mesh overlap is adequate, however, mathematical confirmation has shown that more important defects require greater overlaps to minimize the risk of migration and recurrence [29].

The fixation, when necessary, of these meshes with traumatic devices, such as sutures or tacks, is recognized as a causal factor in the development of postoperative pain and directly related to the damage or entrapment of nerve endings in the receptor tissue, which largely cause postoperative neuralgia in patients after hernia surgery.

Alternatively, either natural or synthetic adhesives applied to the receptor tissue bed are used as a common alternative for mesh fixation [30-32]. Some of these medical adhesives have already been evaluated by our research group grip mesh at 14 days (a-c) and 90 days post-implantation (d-f). Symbols: f mesh filaments; (m) muscle; and (mi) microhook

in preclinical studies with very promising results, especially using long-chain $\mathrm{CA}$, which considerably reduces tissue toxicity and shows quite good biological and mechanical behavior in the hernia repair process [24, 33]. In the present experimental study, we used one of these tissue adhesives, namely, an n-hexyl CA monomer, as a control to compare its biological and mechanical behavior with that of selffixing meshes, which constitute another very good alternative for atraumatic abdominal prosthetic fixation. This group includes meshes equipped with systems that fix the mesh to the receptor tissue either through "grip" type devices [14, $34]$ or through adhesive hydrogels included with the meshes [13, 16, 27, 28, 35, 36].

We designed a biological and mechanical study of the behavior of the two self-fixing meshes and selected two prostheses with different fixation mechanisms, the selfadhesive hydrogel mesh (Adhesix) and the self-gripping (ProGrip®), which both avoid the use of additional fixation and have demonstrated a significant reduction in the incidence of postoperative pain at the clinical level [26-28].

Most of the published clinical studies have analyzed short-term complications, chronic pain and recurrence rates as their primary outcomes [35, 37, 38]. However, few 

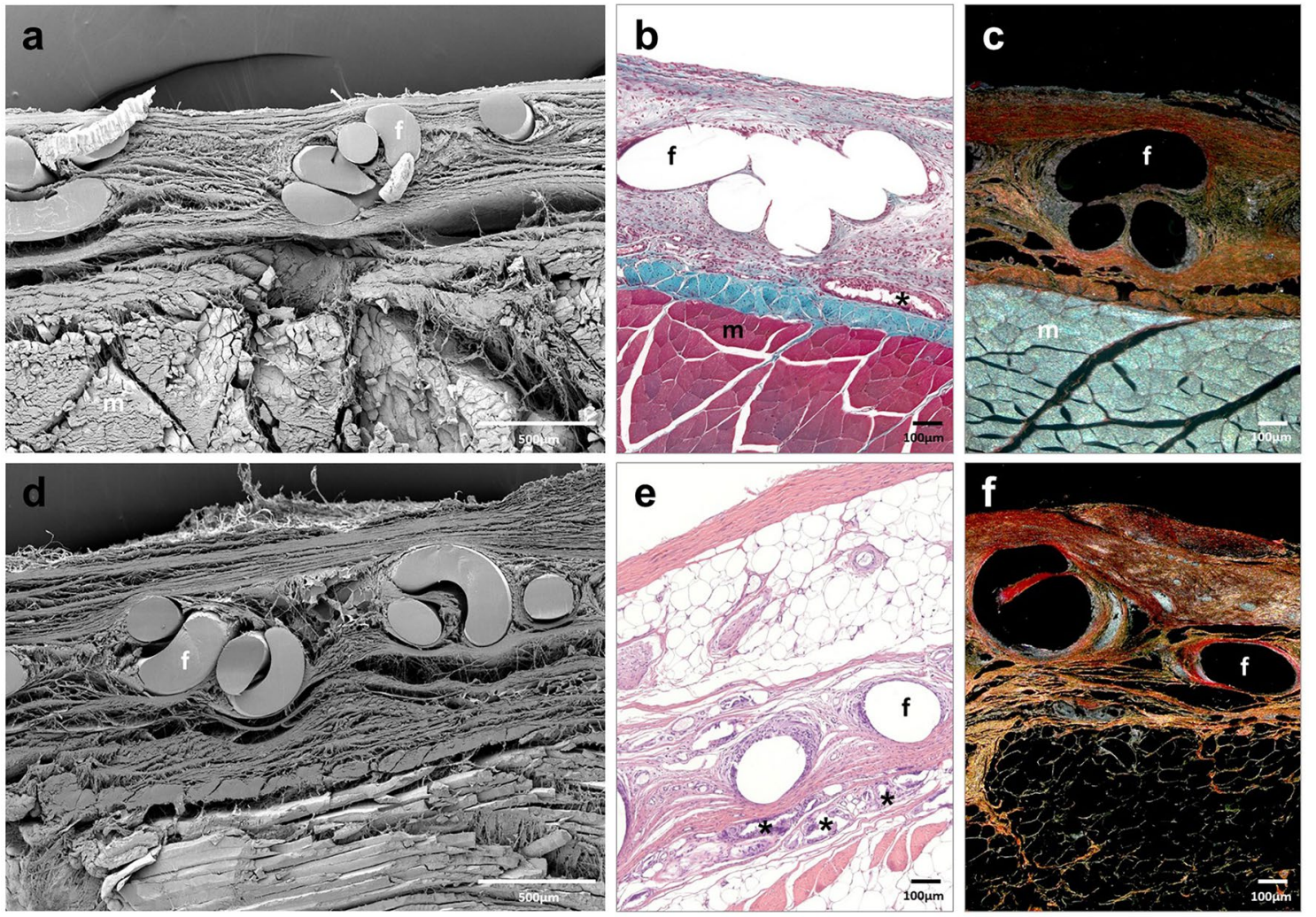

Fig. 5 Scanning electron microscopy $(\mathbf{a}, \mathbf{d})(\times 500$; scales: $500 \mu \mathrm{m})$ and light microscopy images (Masson trichrome (b), hematoxylin and eosin (e) and Sirius red staining $(\mathbf{c}, \mathbf{f}), \times 100$; scale: $100 \mu \mathrm{m})$ of Sur-

publications, none of them related to Adhesix, have examined the mechanical behavior and host tissue response in preclinical animal models using the aforementioned selffixing devices. In our study, the experimental animal was the New Zealand white rabbit, for which we have extensive experience [39]. The experimental model is an acute partial abdominal wall defect with a sufficient size to carry out both morphological and mechanical studies. The study times of 14 and 90 days were designed to corroborate the short- and medium-term behavior of the materials used, with special emphasis on their mechanical response.

At the macroscopic level, we observed that in the short term, Progrip and Surgipro CA showed good integration within the host tissue versus Adhesix, with 4 of the 6 implants showing seroma and 3 implants exhibiting mesh that appeared detached from the recipient tissue, which explained why this study time could not be included in the biomechanical studies and only the medium term of 90 days was included. These indications suggest that fixation with the resorbable hydrogel formed by polyethylene glycol and polyvinylpyrrolidone is not strong enough and impairs the tissue integration process. Indeed, if the hydrogel interferes by obstructing the pores of the mesh, can prevent tissue gipro CA mesh at 14 days (a-c) and 90 days post-implantation (d-f). Symbols: f mesh filaments; (m) muscle; and (*) tissue adhesive

integration of the prosthetic material. In the case of the Progrip this does not happen, that is why it is the one that best integrates into the receptor tissue. Prospective clinical studies have shown low rates of postoperative complications following inguinal hernia repair with Adhesix, which is inconsistent with our results [35, 40]. A 3-year retrospective study also showed that this mesh is associated with low chronic pain, recurrence and postoperative complication rates [27].

In contrast, experimental studies in rats have shown that this hydrogel fixation mesh has a greater probability of dislocation from its implantation site than mechanical grip fixation, which is consistent with our results [41], and such dislocation would translate into recurrences in clinical practice. The lack of integration in Adhesix was also observed in our case three months after implantation in 2 of the 6 samples. Good adhesion of Progrip and Surgipro CA was observed in this same study time, and no presence of seroma was seen in either group.

Our implants also showed a significantly higher degree of mesh shrinkage in the Adhesix group than in the rest of the groups both in the short and long term, reaching important values close to $30 \%$ at 90 days. Previous authors [41] have also shown this problem of prosthetic 
Fig. 6 Macrophage response. Micrographs representative of Adhesix (a, d), Progrip (b, e) and Surgipro CA $(\mathbf{c}, \mathbf{f})$ meshes, demonstrating the distribution of RAM-11-positive cells in red around the prosthetic filaments (immunohistochemistry, $\times 200$; scales: $100 \mu \mathrm{m}$ ) at 14 days (a-c) and 90 days (d-f). Symbols: $\mathbf{f}$ mesh filaments; $(\rightarrow)$ labeled macrophages and $(*)$ tissue adhesive. Macrophage-positive cells per field of the different meshes at the different time points $(\mathbf{g}) *(p<0.05)$ and $* *(p<0.01)$
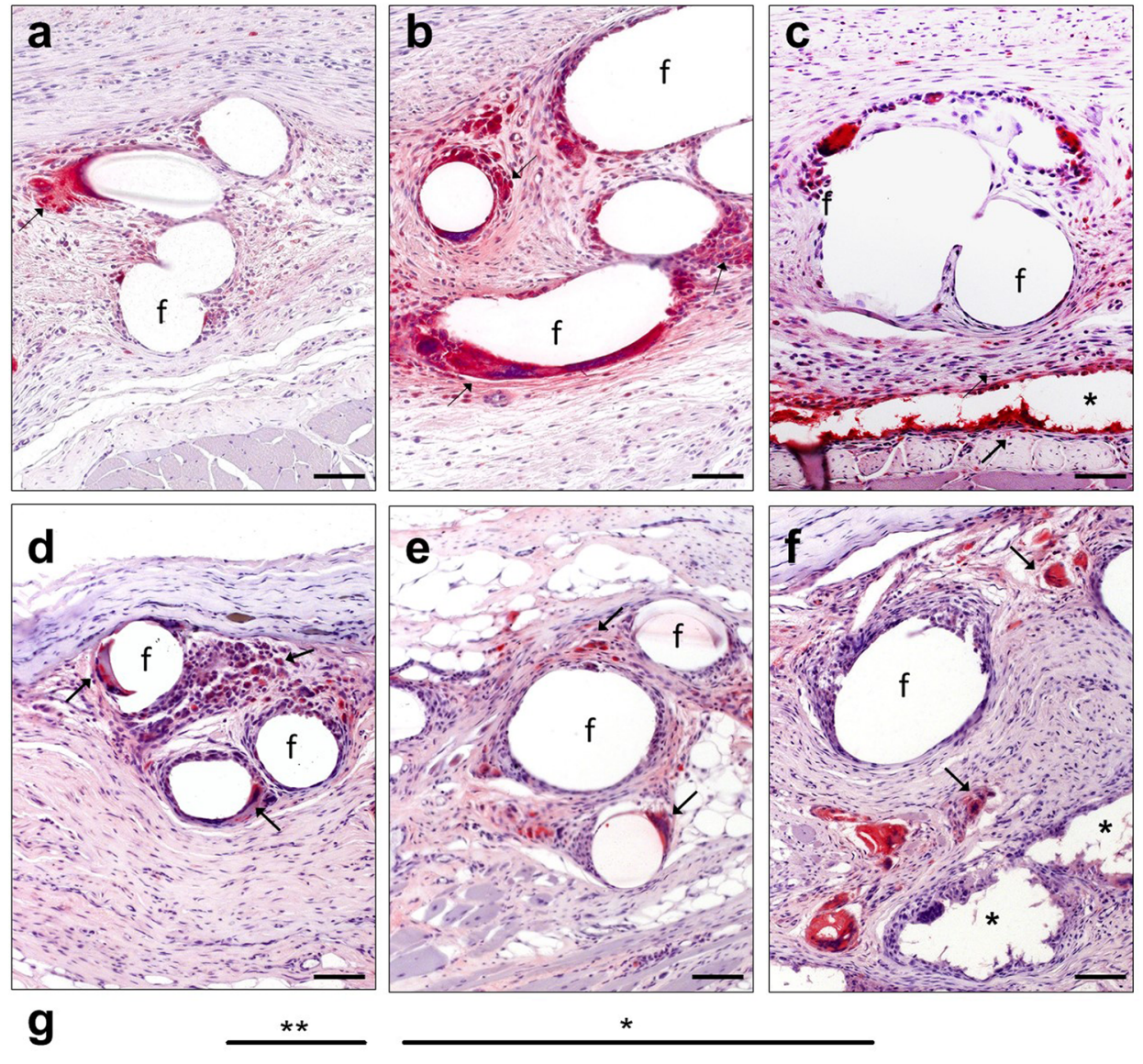

*

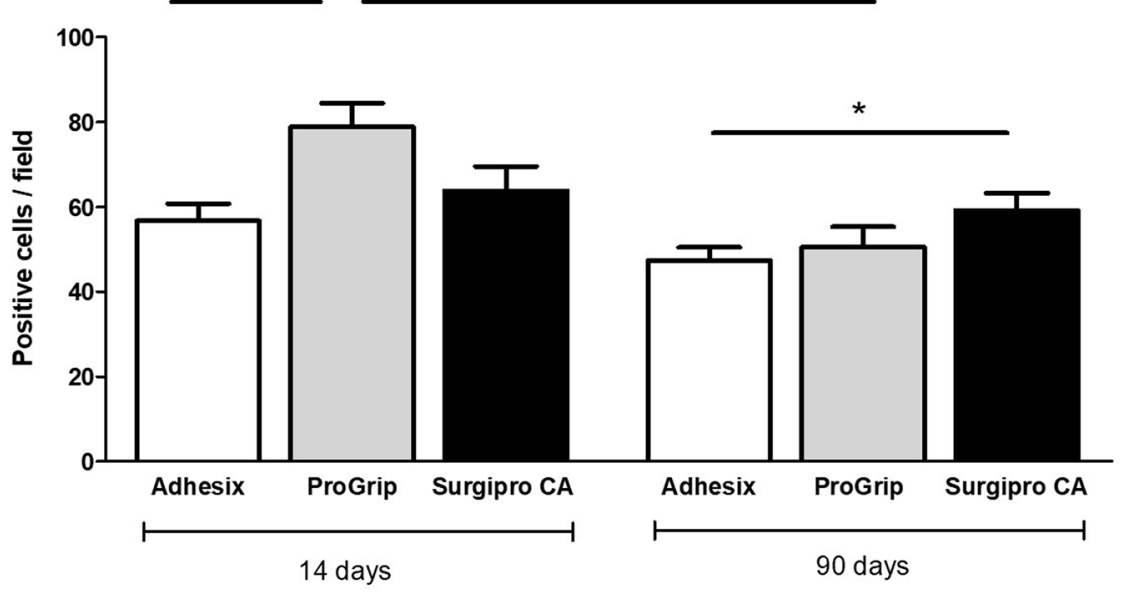

contraction but to a lesser extent. In this case, mesh shrinkage was below statistical significance and PP reached 5\% in some implants, which is still considered not problematic. Incorporation of the Adhesix prosthesis was evaluated as excellent both macroscopically and microscopically in laparoscopic surgery performed in pigs [13], with a significant amount of fibrosis thickness and no real shrinkage; however, $16 \%$ of cases showed mild folding.

Referring to the biomechanical properties, at 90 days, the highest tensile strength values were recorded in the Progrip group and the lowest ones were obtained in the Adhesix group, with strong significant differences observed between both groups. However, the maximum significant value of failure stretch was observed in the Adhesix group compared to the other two groups, thus indicating that the fixation in the Adhesix group was more inefficient, with a higher elongation and a lower tension at failure meaning that the system was very deformable and the fixation was weaker.

Few studies have analyzed the biomechanical properties of self-fixing meshes, and none of these studies have focused on Adhesix. 
Fig. 7 Biomechanical assay sequence showing grip displacement in the lap-shear method (a). Mean tensile strength and failure stretch values $( \pm$ standard deviation) for the different fixation groups at 90 days (b). $*(p<0.05) ; * *(p<0.01)$; and $* * *(p=0.0001)$

a

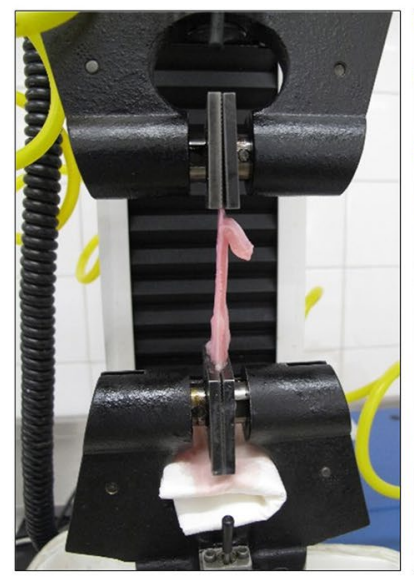

b

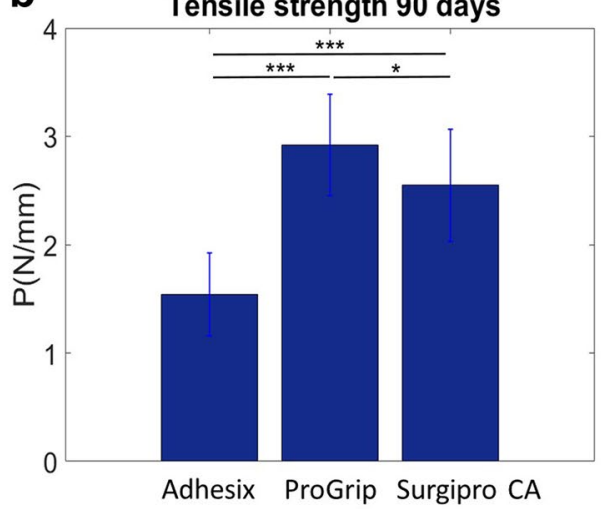

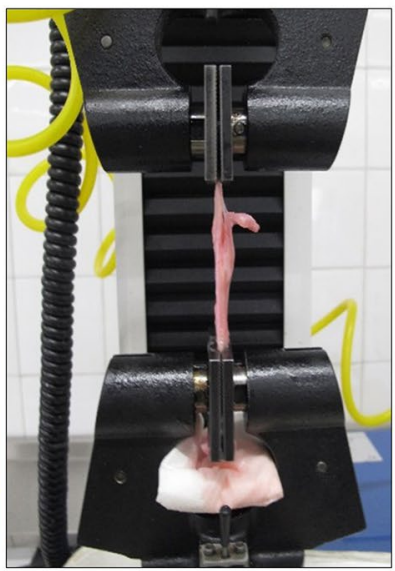

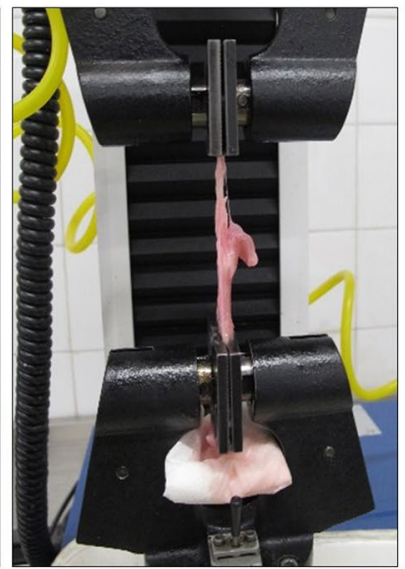

Failure stretch 90 days

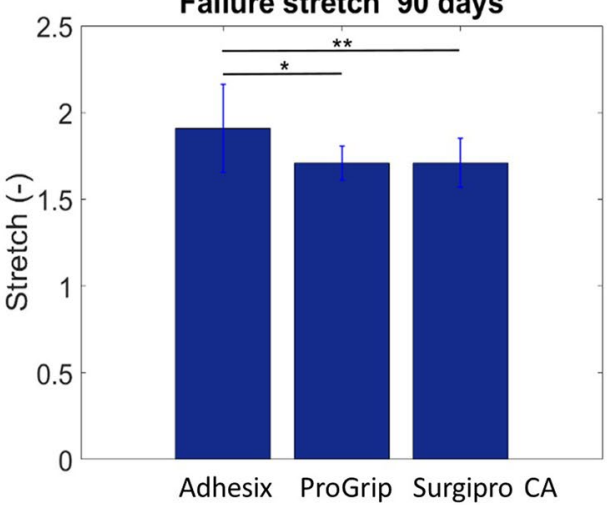

An experimental study confronting Progrip with fixation with a stapler or fibrin glue in a rat model noted that the self-fixing prosthesis showed substantially stronger strength of incorporation in muscle tissue compared with the other fixation systems both in the short and medium term at 5 days and 2 months, respectively [34]. Another experimental study [7] on mongrel swines that compared fibrin sealant and Progrip with a self-fixing mesh also made of PP coated with gelatin, which undergoes intra- and intermolecular crosslinking catalyzed by microbial transglutaminase (LifeMesh ${ }^{\mathrm{TM}}$ ) after contact with water, showed unexpected results for this mesh, which that significantly increased the strength of the mesh-fascia interface. Biomechanical testing in this case was performed $10 \mathrm{~min}$ after implantation of the mesh, which is considered a very early time point that does not allow for tissue growth and prosthetic integration. The authors explain that this crosslinking gelatin technology represents a relatively strong adhesive [7].

An ex vivo porcine and bovine model used to assess gelcoated ProGrip for dislodgement shear forces before and after dissolving the gel showed that this technology significantly decreased the attachment forces of the ProGrip mesh and did not impair the self-gripping properties after dissolving [42].
Regarding the histological examination in our study, the Adhesix samples corroborated the macroscopic observations regarding the lack of integration of the mesh and the presence of seroma below the prosthetic material. The rest of the meshes presented good integration with healing tissue that matured in the long term, was richer in type I collagen and had an increased adipose component. The complete disappearance of the adhesive hydrogel of the Adhesix was observed at 14 days, while the hooks of the Progrip were still present, which led to a higher macrophage response in this last study group. Microhooks are reabsorbed within 12-15 months, whereas the hydrogel was reabsorbed within 7 days of implantation. Other authors [34] have histologically corroborated that the microhooks of the self-gripping mesh were also generously surrounded by tissue and embedded to a depth of approximately $0.5 \mathrm{~mm}$ in the deeper tissue. This superior tissue integration was reflected in the biomechanical results, with the highest tensile strength values recorded in the Progrip group [34].

The Progrip mesh showed the greatest macrophage response in the short term; however, in the long term, RAM11-positive cells decreased considerably in all of our study groups, with a significant decrease in the Progrip group, although the microhooks of resorbable material were still 
present at this time of study. Hollinsky et al. [34] also showed that the inflammatory reaction in Progrip was considerably more severe in the short term than after 2 months and observed fewer lymphoid, plasma, macrophage and granulocyte cells in the repair tissue, and these findings are consistent with our results. Histological examinations of other studies comparing the two self-fixing meshes included here have also found a slightly pronounced foreign body reaction represented by macrophages and foreign body giant cells in all groups [41]. Other experimental studies that have histologically observed the effects of Adhesix [13] have reported that at one week and one month, incorporation of the prosthesis within the abdominal wall was complete in all cases, with the development of a fibrous and inflammatory reaction limited to the mesh and its close periphery; however, none of these studies showed corresponding images.

Obviously, certain limitations were observed in our study. For example, the animal model cannot be easily transferred to the clinic and may not be generalized to the human population. Thus, although good results were shown by these two self-fixing meshes in the clinical setting, conclusions about the behavior of these materials should be made with caution. The high seroma index found in Adhesix could be related to tissue manipulation and the existence of areas where the self-adhering gel coating had not been activated by humidity and body heat; moreover, the absorbance of water from the adjacent tissue and loss of adhesive properties in these areas could have translated into a lack of integration due to mesh displacement and shrinkage [43]. Another limitation that should be taken into consideration is that the onlay model can allow for some movement of the mesh [41].

Taking into account all these considerations and the results obtained in our study, we can conclude that PP meshes with mechanical microgrip self-fixation show better biological and mechanical behavior than hydrogel fixation in our model of abdominal hernia repair in rabbits.

Acknowledgements The study was supported by a Grant SAF201789481-P and PDC2021-121809-I00 from the Spanish Ministry of Science, Innovation and Universities. Financial support from the CIBER$\mathrm{BBN}$ is acknowledged.

Funding Open Access funding provided thanks to the CRUE-CSIC agreement with Springer Nature. The study was supported by Grant SAF2017-89481-P and PDC2021-121809-I00 from the Spanish Ministry of Science and Innovation.

Availability of data and material Data presented in this study are available in the manuscript.

Code availability Not applicable.

\section{Declarations}

Conflict of interest The authors declare that they have no conflict of interest.

Ethics approval Study was conducted according to the guidelines of the Declaration of Helsinki and approved by the Animal Experimentation Ethics Committee of the University of Alcala ([PROEX 045/18] Madrid, Spain).

Consent to participate Not applicable.

Consent for publication Not applicable.

Human and animal rights The up-keep and handling of animals throughout the study was in accordance with the Guide for the Care and Use of Laboratory Animals of the National and European Institutes of Health (Spanish Law 6/2013, Spanish Royal Decree 53/2013, European Directive 2010/63/UE and European Convention of the Council of Europe ETS123). All procedures were performed at the Animal Research Centre of the Universidad de Alcalá (Madrid, Spain), which is registered with the Directorate General for Agriculture of the Ministry of Economy and Technology Innovation of the Community of Madrid (ES280050001165) ensuring all facilities legally cover the needs and requirements of the research.

Open Access This article is licensed under a Creative Commons Attribution 4.0 International License, which permits use, sharing, adaptation, distribution and reproduction in any medium or format, as long as you give appropriate credit to the original author(s) and the source, provide a link to the Creative Commons licence, and indicate if changes were made. The images or other third party material in this article are included in the article's Creative Commons licence, unless indicated otherwise in a credit line to the material. If material is not included in the article's Creative Commons licence and your intended use is not permitted by statutory regulation or exceeds the permitted use, you will need to obtain permission directly from the copyright holder. To view a copy of this licence, visit http://creativecommons.org/licenses/by/4.0/.

\section{References}

1. Kigsnorth A, LeBlanc K (2003) Hernias: inguinal and incisional. Lancet 362:1561-1571. https://doi.org/10.1016/S0140-6736(03) 14746-0

2. Klinge U, Klosterhalfen B, Ottinger AP, Junge K, Schumpelick $\mathrm{V}$ (2002) PVDF as a new polymer for the construction of surgical meshes. Biomaterials 23:3487-3493. https://doi.org/10.1016/ s0142-9612(02)00070-4

3. Klosterhalfen B, Junge K, Klinge U (2005) The lightweight and large porous mesh concept for hernia repair. Expert Rev Med Dev 2:103-117. https://doi.org/10.1586/17434440.2.1.103

4. Junge K, Rosch R, Krones CJ, Klinge U, Mertens PR, Lynen P, Schumpelick V, Klosterhalfen B (2005) Influence of polyglecaprone 25 (Monocryl) supplementation on the biocompatibility of a polypropylene mesh for hernia repair. Hernia 9:212-217. https://doi.org/10.1007/s10029-004-0315-5

5. Martin DP, Badhwar A, Shah DV, Rizk S, Eldridge SN, Gagne DH, Ganatra A, Darois RE, Williams SF, Tai HC, Scott JR (2013) Characterization of poly-4-hydroxybutyrate mesh for hernia repair applications. J Surg Res 184:766-773. https://doi.org/10.1016/j. jss.2013.03.044 
6. Scott JR, Deeken CR, Martindale RG, Rosen MJ (2016) Evaluation of a fully absorbable poly-4-hydroxibutyrate/absorbable barrier composite mesh in a porcine model of ventral hernia repair. Surg Endosc 30:3691-3701. https://doi.org/10.1007/ s00464-016-5057-9

7. Shahan CP, Stoikes NN, Roan E, Tatum J, Webb DL, Voeller GR (2017) Short-term strength of non-penetrating mesh fixation: LifeMesh $^{\mathrm{TM}}$, Tisseel ${ }^{\mathrm{TM}}$, and ProGrip ${ }^{\mathrm{TM}}$. Surg Endosc 31:1350 1353. https://doi.org/10.1007/s00464-016-5119-z

8. Eriksen JR, Bisgaard T, Assaadzadeh S, Jorgensen LN, Rosenberg J (2011) Randomized clinical trial of fibrin sealant versus titanium tacks for mesh fixation in laparoscopic umbilical hernia repair. $\mathrm{Br}$ J Surg 9:1537-1545. https://doi.org/10.1002/bjs.7646

9. Muysoms FE, Norik B, Kyke-Lienhase I, Berrevoet F (2012) Mesh fixation alternatives in laparoscopic ventral hernia repair. Surg Technol Int 2012:125-132

10. Sanders DL, Nienhuijs S, Ziprin P, Miserez M, Gingell-Little-John M, Smeds S (2014) Randomized clinical trial comparing selfgripping mesh with suture fixation of lighweight polypropylene mesh in open hernia repair. Br J Surg 101:1373-1382. https://doi. org/10.1002/bjs.9598

11. Molegraaf M, Kaufmann R, Lange J (2018) Comparison of selfgripping mesh and sutured mesh in open inguinal hernia repair: a meta-analysis of long-term results. Surgery 163:351-360. https:// doi.org/10.1016/j.surg.2017.08.003

12. van Hanswijck de Jonge P, Lloyd A, Horsfall L, Tan R, O'Dwyer PJ (2008) The measurement of chronic pain and health-related quality of life following inguinal hernia repair: a review of the literature. Hernia 12:561-569. https://doi.org/10.1007/ s10029-008-0412-y

13. Champault G, Polliand C, Dufour F, Ziol M, Behr L (2009) A "self adhering" prosthesis for hernia repair: experimental study. Hernia 13:49-52. https://doi.org/10.1007/s10029-008-0419-4

14. Chastan $P$ (2009) Tension-free open hernia repair using an innovative self-gripping semi-resorbable mesh. Hernia 13:137-142. https://doi.org/10.1007/s10029-008-0451-4

15. De Goede B, Klitsie PJ, Van Kempem BJH, Timmermans L, Jeeckel J, Kazemier G, Lange F (2013) Meta-analysis of glue versus sutured mesh fixation for Lichtenstein inguinal hernia repair. Br J Surg 100:735-742. https://doi.org/10.1002/bjs.9072

16. Ramot Y, Kronfeld N, Steiner M, Klaiman G, Hadid A, Sudak M, Nyska A (2019) Biodegradabiliy and safety study of life-mesh, a novel self-adhesive mesh, in sprague-dawley rats. Toxicol Pathol 47:483-493. https://doi.org/10.1177/0192623319833906

17. Bullen NL, Hajibandeh S, Hjibandeh S, Smart NJ, Antoniou SA (2021) Suture versus self-gripping mesh for open inguinal hernia repair: a systematic review with meta-analysis and trial sequential analysis. Surg Endosc 35:2480-2492. https://doi.org/10.1007/ s00464-020-07658-6

18. Shen Y, Sum W, Chen J, Liu S, Wang M (2012) NBCA medical adhesive (n-butyl-2-cyanoacrylate) versus suture for patch fixation in Linchtenstein inguinal herniorraphy: a randomized controlled trial. Surgery 151:550-555. https://doi.org/10.1016/j.surg.2011. 09.031

19. Kükleta JF, Freytag C, Weber M (2012) Efficiency and safety of mesh fixation in laparoscopic inguinal hernia repair using $n$-butyl cyanoacrylate: long-term biocompatibility in over 1300 mesh fixations. Hernia 16:153-162. https://doi.org/10.1007/ s10029-011-0887-9

20. Chen DC, Amid PK (2014) Prevention of inguinodynia: the need for continuous refinement and quality improvement in inguinal hernia repair. World J Surg 38:2571-2573. https://doi.org/10. 1007/s00268-014-2626-8

21. Bjurstrom MF, Nicoli AL, Amid PK, Chen DC (2014) Pain control following inguinal herniorrhaphy: current perspectives. J Pain Res 7:277-290. https://doi.org/10.2147/JPR.S47005
22. Negro P, Basile F, Brescia A, Buonanno GM, Campanelli G, Canonico $S$ et al (2011) Open tension-free Lichtenstein repair of inguinal hernia: use of fibrin glue versus sutures for mesh fixation. Hernia 15:7-14. https://doi.org/10.1007/s10029-010-0706-8

23. Campanelli G, Pascual MH, Hoeferlin A, Rosenberg J, Champault G, Kingsnorth A, Miserez M (2012) Randomized, controlled, blinded trial of Tisseel/Tissucol for mesh fixation in patients undergoing Lichtenstein technique for primary inguinal hernia repair: results of the TIMELI trial. Ann Surg 255:650-657. https:// doi.org/10.1097/SLA.0b013e31824b32bf

24. Pascual G, García-Moreno F, Pérez-Köhler B, Rodríguez M, Benito-Martínez S, Bellón JM (2020) Mesh fixation using a cyanoacrylate applied as a spray improves abdominal wall tissue repair. J Surg Res 246:26-33. https://doi.org/10.1016/j.jss.2019.08.020

25. Leggat PA, Smith DR, Kedyarune U (2007) Surgical applications of cyanoacrylate adhesives: a review of toxicity. ANZ J Surg 77:209-213. https://doi.org/10.1111/j.1445-2197.2007.04020.x

26. Verhelst J, de Goede B, Kleinrensink GJ, Jeekel J, Lange JF, van Eeghem K (2015) Open incisional hernia repair with a self-gripping retromuscular Parietex mesh: a retrospective cohort study. Int J Surg 13:184-188. https://doi.org/10.1016/j.ijsu.2014.11.043

27. Tabbara M, Genser L, Bossi M, Barat M, Polliand C, Carandina S, Christophe Barrat Ch (2016) Inguinal hernia repair using selfadhering sutureless mesh Adhesix: a 3 year follow-up with low chronic pain and recurrence rate. Am Surg 82:112-116

28. Thölix AM, Kössi J, Remes V, Scheinin T, Harju J (2018) Lower incidence of postoperative pain often open inguinal hernia surgery with the usage of synthetic glue-coated mesh (Adhesix). Am Surg 84:1932-1937

29. Tulloh B, de Beaux A (2016) Defects and donuts: the importance of the mesh:defect area ratio. Hernia 20:893-895. https://doi.org/ 10.1007/s10029-016-1524-4

30. Fortelny RH, Petter-Puchner A, Glaser KS, Redl H (2012) Use of fibrin sealant (Tisseel/Tissucol) in hernia repair: a systematic review. Surg Endosc 26:1803-1812. https://doi.org/10.1007/ s00464-012-2156-0

31. Campanelli G, Pascual MH, Hoeferlin A, Rosenberg J, Champault G, Kingsnorth A, Bagot d'Arc M, Miserez M (2014) Postoperative benefits of Tisseel(囚)/Tissucol (®) for mesh fixation in patients undergoing Lichtenstein inguinal hernia repair: secondary results from the TIMELI trial. Hernia 18:751-760. https://doi.org/ 10.1007/s10029-014-1263-3

32. Reynvoet S, Van Cleven I, Van Overbeke K, Chiers P, De Baets $\mathrm{R}$, Troisi F, Berrevoet (2015) The use of cyanoacrylate sealant as simple mesh fixation in laparoscopic ventral hernia repair: a large animal evaluation. Hernia 19:661-670. https://doi.org/10.1007/ s10029-015-1347-8

33. Pascual G, Rodríguez M, Mesa-Ciller C, Pérez-Köhler B, Fernández-Gutiérrez M, San Román J, Bellón JM (2017) Sutures versus new cyanoacrylates in prosthetic abdominal wall repair: a preclinical long-term study. J Surg Res 220:30-39. https://doi.org/ 10.1016/j.jss.2017.06.074

34. Hollinsky Ch, Kolbe T, Walter I, Joachim A, Sandberg S, Koch T, Rülicke T (2009) Comparison of a new self-gripping mesh with other fixation methods for laparoscopic hernia repair in a rat model. J Am Coll Surg 208:1107-1114. https://doi.org/10.1016/j. jamcollsurg.2009.01.046

35. Champault G, Torcivia A, Paolino L, Chaddad W, Lacaine F, Barrat C (2011) A self-adhering mesh for inguinal hernia repair: preliminary results of a prospective multicenter study. Hernia 15:635-641. https://doi.org/10.1007/s10029-011-0843-8

36. Yehuda AB, Nyska A, Szold A (2019) Mesh fixation using a novel bio-adhesive coating compared to tack fixation for IPOM hernia repair: in vivo evaluation in a porcine model. Surg Endosc 33:2364-2375. https://doi.org/10.1007/s00464-019-06806-x 
37. Kroese LF, van Eeghem LHA, Verhelst J, Jeekel J, Kleinrensink GJ, Lange JF (2017) Long term results of open complex abdominal wall hernia repair with self-gripping mesh: a retrospective cohort study. Int J Surg 44:255-259. https://doi.org/10.1016/j.ijsu. 2017.07.029

38. Zhang C, Li F, Zhang H, Zhong W, Shi D, Zhao Y (2013) Selfgripping versus sutured mesh for inguinal hernia repair: a systematic review and meta-analysis of current literature. J Surg Res 185:653-660. https://doi.org/10.1016/j.jss.2013.07.035

39. Bellón JM, Rodríguez M, Pérez-Khöler B, Pérez-López P, Pascual G (2017) The New Zealand white rabbit as a model preclinical studies addressing tissue repair at the level of the abdominal wall. Tissue Eng Part C Methods 23:863-880. https://doi.org/10.1089/ ten.TEC.2017.0167

40. Tollens T, Kennes J, Vermeiren K, Aelvoet C (2014) Prospective, single center, single surgeon's experience with an atraumatic self-adhering mesh in 100 consecutive patients. Surg Technol Int $24: 178-182$

41. Gruber-Blum S, Riepl N, Brand J, Keibl C, Redl H, Fortelny RH, Petter-Puchner AH (2014) A comparison of Progrip(®) and Adhesix (®) self-adhering hernia meshes in an onlay model in the rat. Hernia 18:761-769. https://doi.org/10.1007/ s10029-014-1258-0

42. Nevler A, Gutman M, Lebedyev A (2014) Assessment of gelcoated delayed self-gripping mesh. JSLS 18(4):e2014.001154. https://doi.org/10.4293/JSLS.2014.001154

43. Tabbara M, Barrat C (2015) Comment to "A comparison of Progrip ${ }^{\circledR}$ and Adhesix ${ }^{\circledR}$ self-adhering hernia meshes in an onlay model in the rat" Gruber-Blum S, Riepl N, Brand J, Keibl C, Redl H, Fortelny RH, Petter-Puchner AH (doi:10.1007/s10029014-1258-0). Hernia 19:535-536. https://doi.org/10.1007/ s10029-015-1366-5

Publisher's Note Springer Nature remains neutral with regard to jurisdictional claims in published maps and institutional affiliations. 